\title{
Angiogenesis and experimental hepatic fibrosis
}

\author{
Queli Teixeira Lemos, Zilton A Andrade/ ${ }^{+}$ \\ Laboratório de Patologia Experimental, Centro de Pesquisa Gonçalo Moniz-Fiocruz, Salvador, BA, Brasil
}

\begin{abstract}
Angiogenesis is a basic change occurring during repair by granulation tissue. This process seems to precede fibrosis formation in most types of chronic liver disease. To examine its presence and significance in different types of hepatic insults, this paper sought to identify the presence, evolution and peculiarities of angiogenesis in the most common experimental models of hepatic fibrosis. The characterization of cells, vessels and extracellular matrix and the identification of factors associated with endothelium (factor VIII RA), vascular basement membrane, other components of the vascular walls (actin, elastin) and the presence of the vascular-endothelial growth factor were investigated. The models examined included Capillaria hepatica septal fibrosis, whole pig serum injections, carbon tetrachloride administration, main bile duct ligation and Schistosoma mansoni infection. The first four models were performed in rats, while the last used mice. All models studied exhibited prominent angiogenesis. The most evident relationship between angiogenesis and fibrosis occurred with the $\mathrm{C}$. hepatica model due to circumstances to be discussed. Special attention was paid to the presence of pericytes and to their tendency to become detached from the vascular wall and be transformed into myofibroblasts, which is a sequence of events that explains the decisive role angiogenesis plays in fibrosis.
\end{abstract}

Key words: angiogenesis - experimental hepatic fibrosis - liver

A relationship between the proliferation of blood vessels (angiogenesis) and hepatic fibrosis has long been suspected. McGee and Patrick (1972) previously stated that "granulation tissue", instead of "collapse", precedes fibrosis formation following massive hepatic-cell necrosis. Impressed by the presence of a large number of blood vessels in cirrhosis, Rappaport et al. (1983) referred to the presence of "vascular-fibrotic septa" in hepatic cirrhosis leading to the abnormal vascular derangement characteristic of that condition. However, the subject gained a more firm basis when the close relationship between the presence of a strong angiogenic-stimulating factor [vascular-endothelial growth factor (VEGF)] and blood vessel proliferation during fibrosis formation was demonstrated in rats following main bile-duct ligation (Rosmorduc et al. 1999). More recently, Maria De Souza et al. (2006) took advantage of a new experimental model of hepatic fibrosis, which appears in $100 \%$ of rats infected with the helminth Capillaria hepatica (Ferreira \& Andrade 1993). In this model, the very early development of fibrosis can be easily detected. This model has been used to demonstrate, through a combination of techniques, that vascular proliferation is not only prominent but also precedes collagen deposition during the formation of new fibrous septa. As a matter of fact, a recent editorial suggested that the use of anti-angiogenic drugs could become a new therapy for the treatment of chronic liver diseases (Lai \& Adams 2005).

Financial support: QTL is a CNPq post-doctoral fellowship recipient. +Corresponding author: zilton@bahia.fiocruz.br

Received 16 October 2009

Accepted 12 May 2010
To better evaluate the real significance of the participation of angiogenesis in hepatic fibrogenesis, the present investigation evaluated the time course of both angiogenesis and fibrosis in experimental models of hepatic fibrosis.

\section{MATERIALS AND METHODS}

Five representative models of hepatic fibrosis were selected. The first four models were performed in rats and the last was performed in mice. In these models, fibrosis was induced by the following methods: (i) repeated pig serum injections (Paronetto \& Popper 1966), (ii) C. hepatica infection (Ferreira \& Andrade 1993), (iii) repeated carbon-tetrachloride $\left(\mathrm{CCl}_{4}\right)$ injections (Myren et al. 1989), (iv) main bile-duct ligation (Gressner 1991) and (v) chronic infection with Schistosoma mansoni (Warren 1966).

Healthy adult male and female Wistar rats weighing approximately $200-250 \mathrm{~g}$ were separated by sex and maintained in large metallic boxes with free access to a commercial balanced diet and water. They were maintained in a well-ventilated room at a temperature of approximately $26^{\circ} \mathrm{C}$. Animals were housed two of the same sex per box. Each experimental group contained 15 animals: 10 were used for the experimental treatment and five as saline-injected controls.

The schistosomiasis experiments included 20 infected young Swiss Webster mice of both sexes (18-20 g) and 10 non-infected mice that served as controls. Prior to animal sacrifice or during surgery, the animals were given intraperitoneal anaesthesia with a mixture of ketamine $(2.5 \mathrm{~mL})$ and xylazine $(0,5 \mathrm{~mL})$ in saline $(1 \mathrm{~mL})$.

\section{Experimental groups}

Pig serum model - Rats were treated with two intraperitoneal injections per week with $1 \mathrm{~mL}$ of total pig serum for 16 weeks for a total of 32 injections. Controls were injected with $1 \mathrm{~mL}$ saline. 
C. hepatica infection - Rats were infected with 800 C. hepatica embryonated eggs administered by a gastric tube. Parasite eggs were obtained, counted and purified as previously reported by Ferreira \& Andrade (1993). Two infected animals and one control were killed and examined 20, 30, 40 and 50 days after infection.

$\mathrm{CCl}_{4}$ intoxication - Male rats were gavaged with a solution of $\mathrm{CCl}_{4}$ in $8 \%$ mineral oil twice a week for 12 weeks, as per the protocol of Rosa et al. (1991).

Main bile-duct ligation - Under aseptic conditions and anaesthesia, rats underwent a midline laparotomy. Two separate points of the main bile duct were identified and ligated. The bile duct was then severed between ligatures. The animals were killed at intervals of four, seven, 14, 30 and 40 days after surgery.

Experimental schistosomiasis - A total of 50 mice were transcutaneously infected with 50 freshly eliminated $S$. mansoni cercariae obtained from experimentally infected Biomphalaria glabrata. After certifying 50 days later that the animals were passing viable schistosome eggs in the stools, they were sacrificed every five days thereafter, two at a time, together with one control. Thus, early (10-12 weeks) and late (16 weeks) infections were represented.

Procedures - Animals belonging to the experimental groups were killed by exsanguination after anaesthesia and opening of the abdomen, followed by the severing of the aorta. After gross inspection, the liver was removed and submitted to histopathology, immunohistochemistry and immunofluorescence as follows.

Histopathology - Thin slices were placed in $10 \%$ formalin ( $\mathrm{pH}$ 7.4) for at least $24 \mathrm{~h}$. Small blocks of tissue were paraffin embedded and cut into $5 \mu \mathrm{m}$ sections. Sections were stained with hematoxylin and eosin, siriusred for collagen and orcein for elastic fibres.

Immunohistochemistry - Small pieces of the liver were immersed in Tissue-Tek (OCT compound-Miles Inc Diagnostic Division, Elkhart, USA), snap frozen in liquid nitrogen and cut in a cryostat at $-20^{\circ} \mathrm{C}$. The sections were stained for Von Willebrand factor (factor VIII RA) and VEGF, using antibodies obtained from Dako. The sections were treated with $10 \%$ poly-L-lysine, fixed in dehydrated acetone and treated with phosphate buffered saline (PBS) containing $0.1 \%$ saponin and $1 \%$ bovine serum albumin (BSA). Non-specific binding was blocked by treating sections with $10 \%$ skim milk in PBS for $20 \mathrm{~min}$ at room temperature (RT). The slides were then incubated with the secondary antibody, sheep anti-Rat IgG conjugated to peroxidase [Dako EnVision ${ }^{\mathrm{TM}}$ System - labelled polymer (Dakopatts, Denmark)], at 1:1000 for $30 \mathrm{~min}$ at $37^{\circ} \mathrm{C}$ in a humidified chamber. Endogenous peroxidases were blocked with $0.3 \% \mathrm{H}_{2} \mathrm{O}_{2}$ for $30 \mathrm{~min}$ at RT. The colour was developed with $0.06 \% 3,3$ '-diaminobenzidine tetrahydrochloride and $0.06 \% \mathrm{H}_{2} \mathrm{O}_{2}$ plus $1 \%$ dimethylsulphoxide. Sections were counterstained with $1 \%$ methyl-green for two min, dehydrated and mounted with Permount. Control sections were included in which primary antibody was either omitted or replaced by normal rat serum.

SM- $\alpha$ actin (mouse anti-SM- $\alpha$ actin, clone 1A4, Dako) was examined in paraffin sections coated with poly-lysine. Antigen retrieval was accomplished through microwave treatment in citrate buffer ( $\mathrm{pH} 6.0)$.

Sections were incubated with the primary antibodies overnight at $4^{\circ} \mathrm{C}$ in a humidified chamber. Primary antibodies were diluted in 2\% BSA in PBS ( $\mathrm{pH} 7.4$ ). After washing in PBS, sections were incubated in $10 \%$ skim milk for 20 min to block non-specific binding.

Immunofluorescence - Cryostat liver sections were stained for collagen type IV, fibronectin and laminin (Institute Merrieux, Lyon, France) using immunofluorescence. The antibodies were raised in rabbits. Primary antisera were diluted 1:40 through 1:100 in PBS. The secondary antibody was a fluoresceinated anti-rabbit IgG (Sigma, St. Louis, MO, USA), diluted 1:50 in a weak solution of Evans blue. Control sections were incubated with saline only or with serum from a normal rat.

\section{RESULTS}

Sirius-red-stained collagen fibres (Figure A) appeared prominent and progressively increased with time in all livers examined, regardless of the experimental model used. Blood vessel proliferation was also present following the early appearance of fibrosis, as seen in histological sections, especially when the vascular basement membrane was depicted by immunofluorescent preparations using anti-laminin and anti-type IV collagen antibodies (Figure B). The presence of focal interstitial VEGF staining was also a constant finding for all models, though it was more evident at the earliest stages of fibrosis development (Figure C).

The models studied differed by the location of the fibrosis and by its intensity. Mouse schistosomiasis resulted in two different lesions: (i) isolated periovular granulomas and periportal fibrosis in the presence of confluent granulomas and (ii) a marked proliferation of blood vessels took place in peri-granulomatous tissue. In the bile-duct ligation model, angiogenesis appeared to be associated with ductular proliferation. This latter presented a marked concentric proliferation of actin-positive periductular cells, which behave like pericytes around proliferated blood vessels (Figure D). The $\mathrm{CCl}_{4}$ models showed two different areas of vascular proliferation and fibrosis formation: periportal and pericentral zones, the former becoming more prominent with time. The $C$. hepatica and the bile-duct ligation models had by far the most evident vascular proliferation in relation to fibrosis formation. In these two models, the microscopic findings of vascular proliferation coupled with the presence of actin-containing cells (pericytes, myofibroblasts) were much more evident at the earlier stages than extra-cellular matrix (collagen) deposition. However, this relationship reversed progressively with passing time. Careful analysis of the microscopic findings did reveal that the same sequence of changes was indeed present with the other models studied, although at a slower pace.

\section{DISCUSSION}

The findings from the present paper agree with the suggestion that the accumulation of excess extra-cellular matrix (fibrosis) in the organism invariably results from the same general mechanism of repair and exhib- 

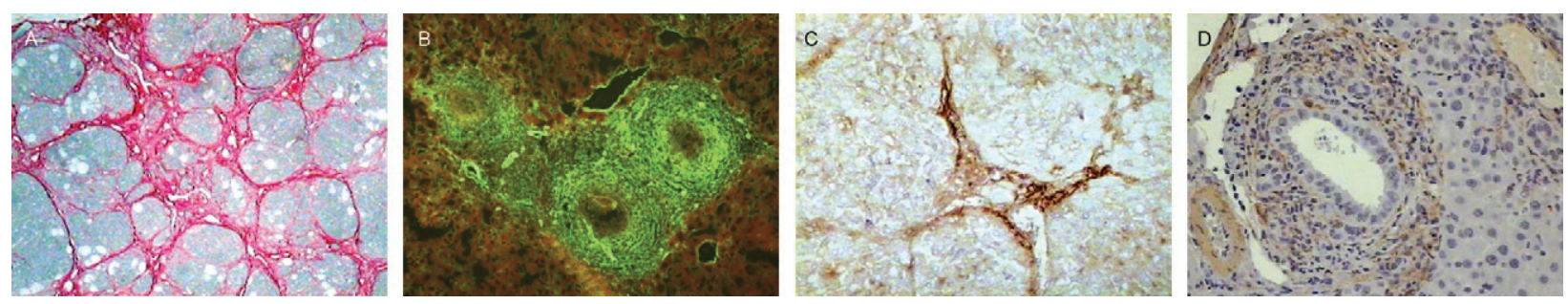

A: carbon-tetrachloride-induced cirrhosis in the rat. The Sirius-red stain reveals collagen in the septa delimitating liver-cell regenerative nodules. Picro-sirius method (200X); B: immunofluorescence for laminin helps the demonstration of the basement membranes of numerous small blood vessels (angiogenesis) disposed around Schistosoma mansoni periovular granulomas in the murine liver, in spite of the non-specific background staining (100X); C: immunohistochemical demonstration for vascular-endothelial growth factor appears as a strong dark-brown staining of the fibrous septa present in this Capillaria hepatica-induced septal fibrosis of the rat liver (200X); D: numerous actin-positive cells are concentrically distributed around a bile duct by the 4th day following surgical ligation of the main biliary duct of the rat. Immunohistochemical stain for SMA (200X).

its both granulation tissue (angiogenesis) and the proliferation of matrix-secreting cells. Several studies have demonstrated that pericytes located within the basement membrane of proliferated capillaries are prone to detach and accumulate within the injured tissues, where they undergo phenotypic transformation into myofibroblasts and become actively engaged in synthesising components of the extra-cellular matrix (Lee et al. 2007). A special emphasis has been placed on the role of hepatic perisinusoidal stellate cells as the most important type of pericyte engaged in hepatic fibrogenesis (Ramadori \& Saile 2004). Recently, doubts have been raised about this concept (Sancho-Bru et al. 2005). Some studies have indicated that several cell types, in addition to the parasinusoidal stellate cells, may participate in hepatic fibrogenesis (Guyot et al. 2006). Using different experimental models of hepatic fibrosis, we have demonstrated angiogenesis and pericyte proliferation at different sites, including the periportal and central acinar zones. These data strongly suggest that other mesenchymal cells, in addition to perisinusoidal cells, are involved in this process. No evidence was found for the participation of stellate cells in fibrogenesis in $C$. hepatica-induced septal fibrosis of the rat liver (Fonseca et al. 2005). The present investigation also demonstrated the presence of actincontaining, pericyte-like periductular cells, in addition to angiogenesis-associated pericytes, in rats with biliary fibrosis induced by main bile duct ligation. On the other hand, perisinusoidal thickening and fibrosis, whenever present, is probably associated with the neighbouring stellate cells, as they possess a high potential for fibrosis formation (Friedman 2008).

Clinical trials began testing anti-angiogenesis medications for the treatment of hepatic fibrogenesis in the wake of studies demonstrating the importance of angiogenesis in hepatic fibrogenesis, in both experimental conditions (Kalluri \& Sukhatme 2000, Corpechot et al. 2002) and human pathology (Bertolini et al. 2001). In a recent editorial, Lai and Adams (2005) suggested that anti-angiogenesis drugs would become a rational choice for the treatment of chronic liver diseases in the near future. In our laboratory, a trial with a supposed anti-angiogenesis drug, thalidomide, failed to interfere with either angiogenesis or fibrosis in mice with experimental schistosomiasis (Lima et al. 2007). However, the indication of anti-angiogenesis drugs for the treatment of liver fibrosis still needs further investigation and it is important to be aware of some pitfalls. The reversal of hepatic lesions following specific treatment for experimental chronic schistosomiasis of mice was studied and demonstrated vascular lesion remodelling with the degradation of excess fibrous tissue. During this process, a marked vascular proliferation was demonstrated by a combination of techniques, including those obtained by the production of vascular plastic casts (Andrade et al. 2006). These findings suggest that angiogenesis may play a dual role in the pathology of hepatic fibrogenesis. Including the present paper, all available data demonstrate the need to further examine the probable dual role of angiogenesis and pericytes in the formation and remodelling of connective tissues.

\section{ACKNOWLEDGEMENTS}

To Ana Cristina Gonzalez and Elisângela Trindade, for immuno-histochemical technical assistance.

\section{REFERENCES}

Andrade ZA, Baptista AP, Santana TS 2006. Remodeling of hepatic vascular changes after specific chemotherapy of schistosomal periportal fibrosis. Mem Inst Oswaldo Cruz 101 (Suppl. I): 267-272.

Bertolini F, Mingrone W, Alietti A, Ferrucci PF, Cocorocchio E, Peccatori F, Cinieri S, Mancuso P, Corsini C, Burlini A, Zucca E, Martinelli G 2001. Thalidomide in multiple myeloma, myelodysplastic syndromes and histiocytosis. Analysis of clinical results and of surrogate angiogenesis markers. Ann Oncol 12: 987-990.

Corpechot C, Barbu V, Wendum D, Kinnman N, Rey C, Poupon R, Housset C, Rosmorduc O 2002. Hypoxia-induced VEGF and collagen I expressions are associated with angiogenesis and fibrogenesis in experimental cirrhosis. Hepatology 35: 1010-1021.

Ferreira LA, Andrade ZA 1993. Capillaria hepatica: a cause of septal fibrosis of the liver. Mem Inst Oswaldo Cruz 88: 441-447.

Fonseca YO, Lima CB, Santos ET, Andrade ZA 2005. On the presence of hepatic stellate cells in portal spaces. Mem Inst Oswaldo Cruz 100: 289-291.

Friedman SL 2008. Hepatic stellate cells: protean, multifunctional and enigmatic cells of the liver. Physiol Rev 88: 125-172. 
Gressner AM 1991. Liver fibrosis: perspectives in pathobiochemical research and clinical outlook. Eur J Clin Chem Clin Biochem 29: 293-311.

Guyot C, Lepreux S, Combe C, Doudnikoff E, Bioulac-Sage P, Balabaud C, Desmoulière A 2006. Hepatic fibrosis and cirrhosis: the (myo)fibroblastic cell subpopulations involved. Int J Biochem Cell Biol 38: 135-151.

Kalluri R, Sukhatme VP 2000. Fibrosis and angiogenesis. Curr Opin Nephrol Hypertens 9: 413-418.

Lai WK, Adams DH 2005. Angiogenesis and chronic inflammation; the potential for novel therapeutic approaches in chronic liver disease. J Hepatol 42: 7-11.

Lee JS, Semela D, Iredale J, Shah VH 2007. Sinusoidal remodeling and angiogenesis: a new funcion for the liver-specific pericyte? Hepatology 45: 817-825.

Lima CB, Iglesias KB, Andrade ZA 2007. Thalidomide failed to inhibit angiogenesis and fibrosis in hepatic schistosomiasis of the mouse. Mem Inst Oswaldo Cruz 102: 883-885.

Maria De Souza M, Tolentino M Jr, Assis BC, Cristina De Oliveira Gonzalez A, Maria Correia Silva T, Andrade ZA 2006. Pathogenesis of septal fibrosis of the liver. (An experimental study with a new model). Pathol Res Pract 202: 883-889.

McGee JO, Patrick RS 1972. The role of perisinusoidal cells in hepatic fibrogenesis. An electron microscopic study of acute carbon tetrachloride liver injury. Lab Invest 26: 429-440.

Myren J, Bang S, Linnestad P, Stave R, Hanssen LE, Dolva LO, Serck-Hanssen A, Arnesen K, Strømme J, Beraki K, Vagene S 1989.
Liver cell necrosis and regeneration following injections of carbon tetrachloride. Effects of the thyrotropin-releasing hormone and somatostatin. APMIS 97: 334-342.

Paronetto F, Popper H 1966. Chronic liver injury induced by immunologic reactions. Cirrhosis following immunization with heterologous sera. Am J Pathol 49: 1087-1101.

Ramadori G, Saile B 2004. Portal tract fibrogenesis in the liver. Lab Invest 84: 153-159.

Rappaport AM, MacPhee PJ, Fisher MM, Phillips MJ 1983. The scarring of the liver acini (cirrhosis). Tridimensional and microcirculatory considerations. Virchows Arch A Pathol Anat Histopathol 402: 107-137.

Rosa H, Parise ER, Paranhos FR, Braga AL, Freitas AS, Hidalgo AN, Mor MB 1991. Controlled production of cirrhosis in the rat liver. Arq Gastroenterol 28: 39-43.

Rosmorduc O, Wendum D, Corpechot C, Galy B, Sebbagh N, Raleigh J, Housser C, Poupon R 1999. Hepatocellular hypoxiainduced vascular endothelial growth factor expression and angiogenesis in experimental biliary cirrhosis. Am J Pathol 155: 1065-1073

Sancho-Bru P, Bataller R, Gasull X, Colmenero J, Khurdayan V, Gual A, Nicolás JM, Arroyo V, Ginès P 2005. Genomic and functional characterization of stellate cells isolated from human cirrhotic livers. J Hepatol 43: 272-282.

Warren KS 1966. The pathogenesis of "clay-pipe stem cirrhosis" in mice with chronic schistosomiasis mansoni, with a note on the longevity of the schistosomes. Am J Pathol 49: 477-489. 